\title{
An Estimation Algorithm for a Class of Networked Control Systems Using UDP-Like Communication Schemes
}

\author{
Michael Epstein, Ling Shi and Richard M. Murray
}

\begin{abstract}
In this work we consider a class of networked control systems (NCS) when the control signal is sent to the plant via a UDP-like communication protocol. In this case the controller sends a communication packet to the plant across a lossy network, but the controller does not receive any acknowledgement signal indicating the status of the control packet. Standard observer based estimators assume the estimator has knowledge of what control signal is applied to the plant. Under the UDP-like protocol the controller/estimator does not have explicit knowledge whether the control signals have been applied to the plant or not. We present a simple estimation and control algorithm that consists of a state and mode observer as well as a constraint on the control signal sent to the plant. For the class of systems considered, discrete time LTI plants where at least one of the states that is directly affected by the input is also part of the measurement vector, the estimator is able to recover the fate of the control packet from the measurement at the next timestep and exhibit better performance than other naive schemes. For single-input-single-output (SISO) systems we are able to show convergence properties of the estimation error and closed loop stability. Simulations are provided to demonstrate the algorithm and show it's effectiveness.
\end{abstract}

\section{INTRODUCTION}

In recent years networked control systems (NCS) have gained much attention in the research community. Networked control systems are characterized by the presences of communication links in the feedback loop. These communication links introduce delays and losses of information in the feedback loop that can degrade the performance of the control system. The effect of information loss is considered in this paper.

The problem of estimating the state of a dynamical system over a lossy network was considered by Sinopoli, et al [1]. The authors considered a discrete time LTI system corrupted by Gaussian noise and analyzed the performance of the Kalman filter when the sensor measurements are sent to the filter across a lossy network. They gave stability conditions for the expected value of the estimation error covariance that was a function of the instability of the system and the frequency of data dropouts. The pure estimation problem was also investigated in [2], [3]

In [4] Sinopoli et al began looking at closing the loop across lossy networks. They added a lossy network between the controller/estimator and the actuators/plant. They attempted to solve the LQG problem in this framework. In this work they made the implicit assumption that the estimator/controller had direct knowledge about the fate of

Control and Dynamical Systems, California Institute of Technology, Pasadena CA 91125 epstein, shiling, murray deds.caltech.edu the control packet sent to the plant by way of an acknowledgement signal, i.e. a TCP-like protocol. They used this assumption to show that a separation principle holds and that the optimal LQG control is linear with a bounded cost when the percentage of loss is below a threshold.

Sinopoli et al began to consider UDP-like communication protocols, where there is no receive acknowledgement, for the network between the controller/estimator and the acutators/plant in [5], [6]. They show that in this setting the LQG controller is in general nonlinear and cannot, in general, be found in closed form. We investigate a similar problem setting in this paper.

We assume there is perfect communication between the sensors and the estimator/controller so that the measurement data is always available at the estimator. The network connecting the estimator/controller to the actuators/plant uses UDP-like protocols. This setup is summarized in Fig. 1. We will model the system as a Jump Linear System (JLS) and present an estimator algorithm guaranteed to recover the fate of the control packet. The estimator algorithm consists of state and mode observers as well as a constraint on the control signal. We will show how this algorithm can be used with a modified state feedback controller to stabilize the closed loop system.

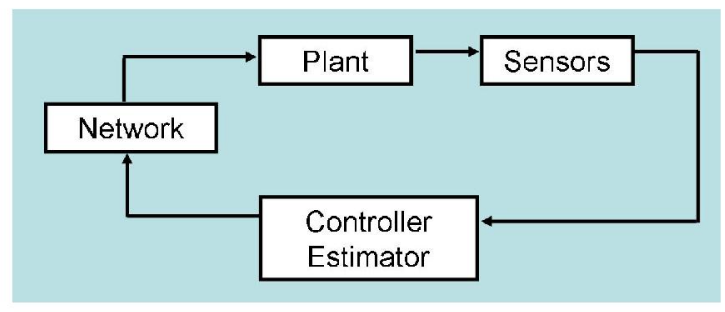

Fig. 1. NCS feedback loop.

Since we will model the system as a JLS it is worthwhile to note some of the estimation literature for JLS. In [7], Alessandri, et al present a receding horizon estimator for a JLS with bounded disturbances. Their problem setting is slightly different in that they consider only open loop JLS, they do not attempt to design a feedback law. The work in [8] designs an optimal compensator for a JLS with Gaussian noise and an unknown switching parameter. Stability conditions are given for the closed loop that appear to be more complicated than those presented here. In [9] Babali and Egerstedt present an algorithm that resembles the one presented in this paper. They also consider a state observer along with a mode detector, however, their algorithm does 
not consider the design of the controller.

The paper is organized as follows. We give an introduction to the problem and related work in Section I. In Section II we set up the problem in a mathematical framework. The estimator algorithm and its convergence properties are presented in Section III. Examples are given in Section IV to illustrate the effectiveness of the algorithm. Finally, conclusions and a description of future extensions to this work are in Section V.

\section{Problem Set UP}

We consider a networked control system where the controller sends commands to the actuator across a packet dropping network as in Fig. 1. The network is assumed to be following UDP-type protocol. That is the controller sends signals to the actuators but does not receive any form of acknowledgement, i.e. the controller does not know if the packet was dropped or not.

The plants we consider are discrete-time linear systems. If the control packet is not received it is assumed the plant applies no control and evolves open loop. The plant is

$$
\begin{aligned}
x_{k+1} & =A x_{k}+\gamma_{k} B u_{k}+w_{k} \\
y_{k} & =C x_{k}+v_{k} \\
\gamma_{k} & \in\{0,1\}
\end{aligned}
$$

where $x \in \mathbb{R}^{n}$ is the state vector, $u \in \mathbb{R}^{r}$ the control input and $y \in \mathbb{R}^{m}$ the sensor output. The process noise is given by $w_{k} \in \mathbb{R}^{n}$ and the measurement noise by $v_{k} \in \mathbb{R}^{m}$. The variable $\gamma_{k}$ indicates if the packet containing $u_{k}$ was received at the plant $\left(\gamma_{k}=1\right)$ or if it was dropped $\left(\gamma_{k}=\right.$ 0 ). It is assumed if the packet is dropped the plant applies no control for that timestep, i.e. it evolves open loop. Note also there is no network between the plant/sensors and the estimator/controller, so measurements are always available. We further assume $A$ is unstable, $(A, B)$ is controllable and $(A, C)$ is observable, so that in the absence of the network $F$ and $L$ are designed to make $A+B F$ and $A-L C A$ stable.

We also make the assumption that $C B \neq 0$. This will be required as we desire to recover the fate of $\gamma_{k}$ at time $k+1$, meaning we need the effect of $\gamma_{k}$ be present in

$$
y_{k+1}=C x_{k+1}=C A x_{k}+\gamma_{k} C B u_{k}+d_{k}
$$

where $d_{k}=C w_{k}+v_{k+1}$. If $C B=0$, the $\gamma_{k}$ would disappear from $y_{k+1}$.

Under the UDP-like communication scheme we are considering, the estimator does not have any knowledge about the value $\gamma_{k}$. Therefore if one were to design an observer for this system it could take the form of

$$
\hat{x}_{k+1}=A \hat{x}_{k}+\hat{\gamma}_{k} B u_{k}+L\left(y_{k+1}-C A \hat{x}_{k}-\hat{\gamma}_{k} C B u_{k}\right)
$$

where the decision must be made how to select $\hat{\gamma}_{k}$.

Writing the estimation error as $e_{k}=x_{k}-\hat{x}_{k}$ we see it evolves according to

$$
e_{k+1}=(A-L C A) e_{k}+\left(\gamma_{k}-\hat{\gamma}_{k}\right)(B-L C B) u_{k}+z_{k}
$$

where we have used the notation $z_{k}=(I-L C) w_{k}-L v_{k+1}$. Let $\mathbf{X}_{k}=\left[x_{k}, e_{k}\right]^{T}$, the closed loop dynamics are

$$
\begin{aligned}
\mathbf{X}_{k+1}= & {\left[\begin{array}{c|c}
A & 0 \\
\hline 0 & A-L C A
\end{array}\right] \mathbf{X}_{k} } \\
& +\left[\begin{array}{c}
\left(\gamma_{k}-\hat{\gamma}_{k}\right)(I-L C)
\end{array}\right] B u_{k}+\left[\begin{array}{c}
w_{k} \\
z_{k}
\end{array}\right]
\end{aligned}
$$

The goal is to design an estimator and a control algorithm that can recover the fate of $\gamma_{k}$ at time $k+1$. It should not require much computation and also stabilize the closed loop in some sense.

\section{A. Naive Schemes}

Before presenting our algorithm, we will first look at several naive schemes for dealing with this situation. To simplify the analysis of these naive schemes we will ignore the noise terms, i.e. let $w_{k}=z_{k}=0$. For the sake of showing the pitfalls of these schemes, it should be clear that if their faults are exposed even in the noise free case they certainly will not be suitable when noise is present. When analyzing our algorithm we will once again include the noise. Let us assume a part of these naive schemes is to include a state feedback controller $u_{k}=F \hat{x}_{k}$.

The closed loop is written as a Jump Linear System (JLS)

$$
\begin{aligned}
\mathbf{X}_{k+1} & =H_{\theta(k)} \mathbf{X}_{k} \\
H_{\theta(k)} & =\left[\begin{array}{c|c}
A+\gamma_{k} B F & -\gamma_{k} B F \\
\hline G_{\hat{\gamma}}(k) & A-L C A-G_{\hat{\gamma}}(k)
\end{array}\right] \\
G_{\hat{\gamma}}(k) & =\left(\gamma_{k}-\hat{\gamma}_{k}\right)(B-L C B) F \\
\gamma_{k}, \hat{\gamma}_{k} & \in\{0,1\}
\end{aligned}
$$

where we see that if $\hat{\gamma}_{k}=\gamma_{k}$ then $G_{\hat{\gamma}}(k)=0$ and the estimation error will evolve as $e_{k+1}=(A-L C A) e_{k}$ which is clearly stable. Recalling the UDP-like communication protocol, however, the estimator receives no acknowledgement and hence does not know the value of $\gamma_{k}$ when deciding on $\hat{\gamma}_{k}$. As a result the estimator could either try to reason about $\gamma_{k}$ (if possible) or simply set $\hat{\gamma}_{k}$ to a predetermined value.

The latter situation is what we refer to as the naive schemes. Let us investigate them and see the drawbacks. If we assume the packet drop sequence is independent and identically distributed (i.i.d) with an expected value given by $\mathbf{E}\left[\gamma_{k}\right]=\bar{\gamma}$, then it might seem logical to set $\hat{\gamma}_{k}=\bar{\gamma}$ for all $k$ as was done in [6]. Though the problem setting was slightly different, the approach can be applied here and the resulting JLS has two modes given by

$$
\begin{gathered}
{\left[\begin{array}{cc}
A+\gamma_{k} B F & \gamma_{k} B F \\
G_{\bar{\gamma}}(k) & A-L C A-G_{\bar{\gamma}}(k)
\end{array}\right],} \\
G_{\bar{\gamma}}(k)=\left(\gamma_{k}-\bar{\gamma}\right)(B-L C B) F,
\end{gathered}
$$

with $\gamma_{k} \in\{0,1\}$. In order for the closed loop to be stable clearly at least one of the switching modes must be stable. When $\gamma_{k}=0$ the state evolves as $x_{k+1}=A x_{k}$ so that mode will be unstable, thus the only hope is if the mode 
corresponding to $\gamma_{k}=1$ is stable. but with only $F$ and $L$ as design parameters, for $\bar{\gamma} \neq 1$ it may not even be possible to make this matrix stable!

Another option for preselecting $\hat{\gamma}_{k}$ is to set it to either 0 or 1 . Clearly setting $\hat{\gamma}_{k}=0$ will not work as in this case none of the modes are stable. If we let $\hat{\gamma}_{k}=1$ then the JLS is defined by

$$
\begin{gathered}
{\left[\begin{array}{cc}
A+\gamma_{k} B F & \gamma_{k} B F \\
G_{1}(k) & A-L C A-G_{1}(k)
\end{array}\right],} \\
G_{1}(k)=\left(\gamma_{k}-1\right)(B-L C B) F,
\end{gathered}
$$

with $\gamma_{k} \in\{0,1\}$. When $\gamma_{k}=1$ we see the switching mode is stable. Thus far it would appear to be the best option to use as an estimator. The downfall is of course, for $\gamma_{k}=0$ the error dynamics are unstable. Any sequence of drops will cause the estimator error to grow. As one would expect, the estimation error can still converge to zero as $k \rightarrow \infty$, but only for values of $\bar{\gamma}$ close to 1 .

For any system we design, the state will evolve open loop when $\gamma_{k}=0$, but if we were able to design a system that could recover $\hat{\gamma}_{k}=\gamma_{k}$ at time $k+1$, then the estimation error would still be converging. In fact, if we can get $\hat{\gamma}_{k}=\gamma_{k}$ we return to the TCP case and can tolerate a higher percentage of drops.

\section{Estimation Algorithm}

As stated above it is clear that it is advantageous to choose an estimator scheme that could recover $\hat{\gamma}_{k}=\gamma_{k}$ as this would make the NCS revert to the TCP-like communication protocol. We will use an estimator algorithm consisting of the state observer from Eqn. (4) and choosing $\hat{\gamma}_{k}$ according to the mode observer

$$
\hat{\gamma}_{k}=\arg \min _{\beta \in\{0,1\}}\left\|y_{k+1}-C A \hat{x}_{k}-\beta C B u_{k}\right\|^{2} .
$$

The norm $\|\cdot\|$ above, and all other norms in this paper, are assumed to be the 2-norm unless otherwise explicitly stated. The mode observer above can be shown to recover the true state of $\gamma_{k}$ under the conditions below.

Proposition 1: The mode observer that chooses $\hat{\gamma}_{k}$ according to Eqn. (11) will give $\hat{\gamma}_{k}=\gamma_{k}$ if

$$
\left|u_{k}^{T} B^{T} C^{T} C B u_{k}\right|>2\left|u_{k}^{T} B^{T} C^{T}\left(C A e_{k}+d_{k}\right)\right|
$$

Proof: Returning to Eqn. (11) we can write

$$
\begin{aligned}
\| y_{k+1}- & C A \hat{x}_{k}-\beta C B u_{k} \|^{2} \\
= & \left\|C A x_{k}+\gamma_{k} C B u_{k}+d_{k}-C A \hat{x}_{k}-\beta C B u_{k}\right\|^{2} \\
= & \left\|C A e_{k}+d_{k}+\left(\gamma_{k}-\beta\right) C B u_{k}\right\|^{2} \\
= & e_{k}^{T} A^{T} C^{T} C A e_{k}+d_{k}^{T} d_{k} \\
& +\left(\gamma_{k}-\beta\right)^{2} u_{k}^{T} B^{T} C^{T} C B u_{k} \\
& +2\left(\gamma_{k}-\beta\right) u_{k}^{T} B^{T} C^{T}\left(C A e_{k}+d_{k}\right)
\end{aligned}
$$

Since $e_{k}^{T} A^{T} C^{T} C A e_{k}+d_{k}^{T} d_{k} \geq 0$ and is independent of $\left(\gamma_{k}, \beta\right)$ we can remove it from the minimization, that leaves

$$
\begin{aligned}
J\left(\gamma_{k}, \beta\right)=\left(\gamma_{k}-\beta\right)^{2} u_{k}^{T} B^{T} C^{T} C B u_{k} \\
\\
+2\left(\gamma_{k}-\beta\right) u_{k}^{T} B^{T} C^{T}\left(C A e_{k}+d_{k}\right) .
\end{aligned}
$$

Recall that $\gamma_{k} \in\{0,1\}$ and $\beta \in\{0,1\}$, so we have four possibilities for $J$ according to Table $\mathbf{I}$.

TABLE I

\begin{tabular}{|c|c|}
\hline$\left(\gamma_{k}, \beta\right)$ & $J$ \\
\hline$(0,0)$ & 0 \\
\hline$(0,1)$ & $u_{k}^{T} B^{T} C^{T} C B u_{k}-2 u_{k}^{T} B^{T} C^{T}\left(C A e_{k}+d_{k}\right)$ \\
\hline$(1,0)$ & $u_{k}^{T} B^{T} C^{T} C B u_{k}+2 u_{k}^{T} B^{T} C^{T}\left(C A e_{k}+d_{k}\right)$ \\
\hline$(1,1)$ & 0 \\
\hline
\end{tabular}

Clearly since $J$ is being minimized, a sufficient condition for $\beta=\gamma_{k}$ is

$$
u_{k}^{T} B^{T} C^{T} C B u_{k} \pm 2 u_{k}^{T} B^{T} C^{T}\left(C A e_{k}+d_{k}\right)>0
$$

and since both terms in Eqn. (13) are scalars this is equivalent to Eqn. (12).

Remark 2: The condition given in Proposition 1 is in terms of the control, error and noise signals. Later it will be shown how this condition can be turned into a design criterion.

Remark 3: The condition given by Eqn. (12) in Proposition 1 is only a sufficient condition to get $\hat{\gamma}_{k}=\gamma_{k}$, it is not necessary. Returning to Table I, we see that for $\gamma_{k}=0$ we only require $u_{k}^{T} B^{T} C^{T} C B u_{k}-2 u_{k}^{T} B^{T} C^{T}\left(C A e_{k}+d_{k}\right)>0$ and for $\gamma_{k}=1$ it is only necessary for $u_{k}^{T} B^{T} C^{T} C B u_{k}+$ $2 u_{k}^{T} B^{T} C^{T}\left(C A e_{k}+d_{k}\right)>0$. Thus the necessary and sufficient conditions for $\hat{\gamma}_{k}=\gamma_{k}$ can be written as

$$
\left\{\begin{array}{l}
\gamma_{k}=0 \text { and } \\
u_{k}^{T} B^{T} C^{T} C B u_{k}-2 u_{k}^{T} B^{T} C^{T}\left(C A e_{k}+d_{k}\right)>0 \\
\quad \text { or } \\
\gamma_{k}=1 \text { and } \\
u_{k}^{T} B^{T} C^{T} C B u_{k}+2 u_{k}^{T} B^{T} C^{T}\left(C A e_{k}+d_{k}\right)>0
\end{array}\right.
$$

but since we don't know the value of $\gamma_{k}$ we use the sufficient condition of Proposition 1.

Lemma 4: If the error has converged and there is no noise, i.e. $e_{k}=0$ and $d_{k}=0$, then the mode observer will return the correct value of $\gamma_{k}$. If $u_{k}=0$ the output of the mode observer does not affect the state observer.

Proof: With $e_{k}=d_{k}=0$ we see that the right hand side of Eqn. (12) is zero as well. Thus for any $u_{k} \neq 0$ the sufficient condition is satisfied and $\hat{\gamma}_{k}=\gamma_{k}$. If $u_{k}=0$ then the left hand side of Eqn. (12) is zero and we could get $\hat{\gamma}_{k} \neq \gamma_{k}$. Of course since $u_{k}=0$ this mistake does not affect the estimation error as $\left(\gamma_{k}-\hat{\gamma}_{k}\right)(B-L C B) u_{k}=0$. 


\section{A. SISO Systems}

It turns out more can be proven if we consider singleinput-single-output (SISO) systems.

Corollary 5: Consider SISO systems, so that $u_{k} \in \mathbb{R}$, $y_{k} \in \mathbb{R}$ and $C B \in \mathbb{R}$ and we can replace Eqn. (12) with

$$
\left|C B u_{k}\right|>2\left|C A e_{k}+d_{k}\right|
$$

Proof: Since we are considering SISO systems we have $C B u_{k} \in \mathbb{R}$ and $\left(C A e_{k}+d_{k}\right) \in \mathbb{R}$. Thus we can write

$$
\begin{aligned}
u_{k}^{T} B^{T} C^{T} C B u_{k} & =\left(C B u_{k}\right)^{2} \\
2 u_{k}^{T} B^{T} C^{T}\left(C A e_{k}+d_{k}\right) & =2\left(C B u_{k}\right)\left(C A e_{k}+d_{k}\right)
\end{aligned}
$$

which we can use to rewrite Eqn. (12) according to

$$
\left|\left(C B u_{k}\right)^{2}\right|>2\left|\left(C B u_{k}\right)\left(C A e_{k}+d_{k}\right)\right|
$$

and simplify to get Eqn. (14).

Now that we have the sufficient condition on $\left(u_{k}, e_{k}, d_{k}\right)$ for $\hat{\gamma}_{k}=\gamma_{k}$, we would like to ensure this sufficient condition is satisfied. If we knew $e_{k}$ and $d_{k}$, we could simply pick $u_{k}$ to satisfy the condition but of course these are unknown. Instead assume we do know a bound on the initial error $\left\|e_{0}\right\| \leq \delta_{e}$ and that we consider only norm bounded noises $\left\|d_{k}\right\| \leq \delta_{d},\left\|z_{k}\right\| \leq \delta_{z}$.

The estimation error from the state observer that is given in Eqn. (5) can be evaluated at time $k$ according to

$$
\begin{aligned}
& e_{k}=(A-L C A)^{k} e_{0}+\sum_{j=0}^{k-1}(A-L C A)^{k-j-1} h_{j} \\
& h_{j}=\left(\gamma_{j}-\hat{\gamma}_{j}\right)(B-L C B) u_{j}+z_{j}
\end{aligned}
$$

If we assume that we are always able to pick a $u_{k}$ satisfying Eqn. 14 then $\hat{\gamma}_{k}=\gamma_{k}$ and the norm of the estimation error can be bounded according to

$$
\left\|e_{k}\right\| \leq\left\|(A-L C A)^{k}\right\| \delta_{e}+M_{k} \delta_{z},
$$

where

$$
M_{k}=\sum_{j=0}^{k-1}\left\|(A-L C A)^{k-j-1}\right\|,
$$

with $M_{0}=0$ and $M_{1}=I$.

Lemma 6: The norm of the estimation error, as given in Eqn. (15), will be bounded as $k \rightarrow \infty$.

Proof: Since $A-L C A$ is stable we have $\lim _{k}\left\|(A-L C A)^{k}\right\|=0$, so the first term in Eqn. (15) will go to zero as $k \rightarrow \infty$. It remains to show that the sum $M_{k}$ will converge and be upperbounded by a finite value, $\lim M_{k}=M<\infty$. Since $A-L C A$ is stable there exists a finite integer $s>0$, such that $K=\left\|(A-L C A)^{s}\right\|<1$. Set $q$ to be the smallest $s$, then for all $i \in[t q,(t+1) q-1)$, $t \geq 1$, we get

$$
\begin{aligned}
\left\|(A-L C A)^{i}\right\| & =\left\|(A-L C A)^{q *(i / q)}\right\| \\
& \leq\left\|(A-L C A)^{q}\right\|^{i / q} \\
& =K^{i / q} \leq K^{t}
\end{aligned}
$$

Therefore we have

$$
\begin{aligned}
M= & \sum_{j=0}^{q-1}\left\|(A-L C A)^{j}\right\|+\sum_{j=q}^{\infty}\left\|(A-L C A)^{j}\right\| \\
= & \sum_{j=0}^{q-1}\left\|(A-L C A)^{j}\right\|+\sum_{j=q}^{2 q-1}\left\|(A-L C A)^{j}\right\| \\
& +\sum_{j=2 q}^{3 q-1}\left\|(A-L C A)^{j}\right\|+\cdots \\
\leq & \sum_{j=0}^{q-1}\left\|(A-L C A)^{j}\right\|+q K+q K^{2}+q K^{3}+\cdots \\
= & \sum_{j=0}^{q-1}\left\|(A-L C A)^{j}\right\|+\frac{q K}{1-K}
\end{aligned}
$$

Hence the estimation error will be bounded as $k \rightarrow \infty$

$$
\lim _{k \rightarrow \infty}\left\|e_{k}\right\| \leq \delta_{z}\left(\sum_{j=0}^{q-1}\left\|(A-L C A)^{j}\right\|+\frac{q K}{1-K}\right)
$$

We can now use this information to design a control law that will ensure $\hat{\gamma}_{k}=\gamma_{k}$ according to the Proposition below.

Proposition 7: For SISO systems, if we pick at each time step a control value that satisfies

$$
\left|u_{k}\right|>\frac{2}{|C B|}\left[\|C A\|\left(\left\|(A-L C A)^{k}\right\| \delta_{e}+M_{k} \delta_{z}\right)+\delta_{d}\right]
$$

then we are guaranteed to have $\hat{\gamma}_{k}=\gamma_{k}$. In addition the right hand side of Eqn. (18) will be remain upperbounded by a finite value as $k \rightarrow \infty$.

Proof: We will prove the first statement by induction. Pick any $u_{0}$ satisfying Eqn. (18). Then we can write

$$
\begin{aligned}
\left|C B u_{0}\right| & >2\left[\|C A\|\left(\left\|(A-L C A)^{0}\right\| \delta_{e}+M_{0} \delta_{z}\right)+\delta_{d}\right] \\
& \geq 2\left[\|C A\|\left\|e_{0}\right\|+\delta_{d}\right] \\
& \geq 2\left[\left\|C A e_{0}\right\|+\delta_{d}\right] \geq 2\left|C A e_{0}+d_{0}\right|,
\end{aligned}
$$

which by Corollary 5 implies $\hat{\gamma}_{0}=\gamma_{0}$ then from Eqn. (15) $\left\|e_{1}\right\| \leq\|A-L C A\| \delta_{e}+M_{1} \delta_{z}$. Now repeat the steps above, pick any $u_{1}$ satisfying Eqn. (18) and we get

$$
\begin{aligned}
\left|C B u_{1}\right| & >2\left[\|C A\|\left\|e_{1}\right\|+\delta_{d}\right] \\
& \geq 2\left[\left\|C A e_{1}\right\|+\delta_{d}\right] \geq 2\left|C A e_{1}+d_{1}\right|,
\end{aligned}
$$

implying $\hat{\gamma}_{1}=\gamma_{1}$ and $\left\|e_{2}\right\| \leq\left\|(A-L C A)^{2}\right\| \delta_{e}+M_{2} \delta_{d}$. Then by induction it can be shown that choosing $u_{k}$ to satisfy Eqn. (18) gives $\hat{\gamma}_{k}=\gamma_{k}$ for all $k$ and the norm of the error stays bounded above by Eqn. (15).

The second statement is a direct result of Lemma 6 . The right hand side of Eqn (18) is upper bounded as $k \rightarrow \infty$ by

$$
\frac{2}{|C B|}\left[\|C A\|\left(\sum_{j=0}^{q-1}\left\|(A-L C A)^{j}\right\|+\frac{q K}{1-K}\right) \delta_{z}+\delta_{d}\right]
$$


We now have a state and mode observer together with a constraint on the control action to ensure the fate of the $k^{\text {th }}$ control packet $\left(\gamma_{k}\right)$ can be recovered at time $k+1$. This assures the estimation error will be bounded. The algorithm is summarized in Fig. 2. We are of course also interested in the closed loop performance of the system. Below we show that in the noise-free case we can achieve almost sure stability with a slightly modified state feedback controller.

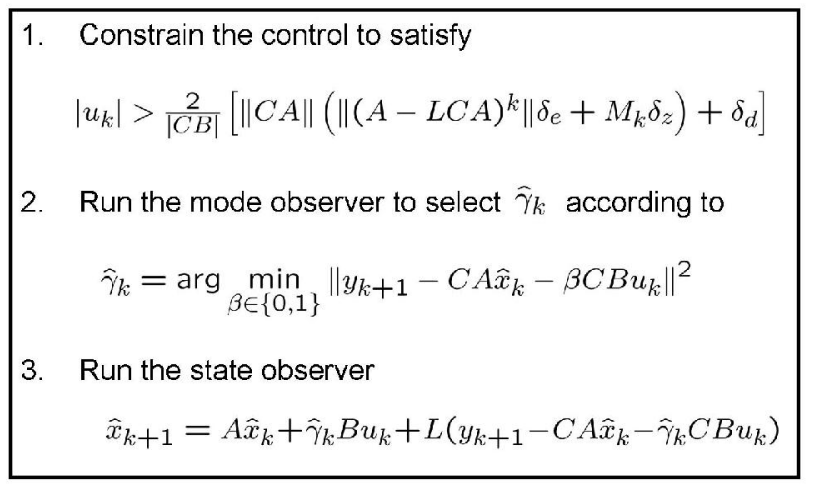

Fig. 2. Estimator Algorithm.

\section{B. SISO and Noise Free Systems}

If we assume there is no noise acting on the system, we have $w_{k}=v_{k}=z_{k}=\delta_{d}=\delta_{z}=0$. In this case the condition of Proposition 7 reduces to

$$
\left|u_{k}\right|>\Delta_{k} \triangleq \frac{2}{|C B|}\|C A\|\left\|(A-L C A)^{k}\right\| \delta_{e} .
$$

As we will now show, using a modified version of a state feedback controller we can make the closed loop system almost surely stable. The definition and sufficient conditions, taken from [10], [11], of almost sure stability for a JLS of the form of Eqn. (7) with $\theta(k) \in\{1,2, \cdots, N\}$ is

$$
\operatorname{Pr}\left[\lim _{k \rightarrow \infty}\left\|\mathbf{X}_{k}\right\|=0\right]=1 \text {. }
$$

If the modes of the JLS, $\{\theta(k)\}$, are a finite-state ergodic Markov process with unique invariant distribution $\operatorname{Pr}[\theta(k)=$ $j]=\pi_{j}$, then the system is almost surely stable if there exists some norm such that $\prod_{i=1}^{N}\left\|H_{i}\right\|^{\pi_{i}}<1$.

Theorem 8: Using the state and mode observers described above along with the state feedback controller

$$
u_{k}=F \hat{x}_{k}+\operatorname{sgn}\left(F \hat{x}_{k}\right) \Delta_{k}
$$

where $F$ is designed such that $A+B F$ is stable, the closed loop system is almost surely stable if for $\bar{\gamma}=\mathbf{E}\left[\gamma_{k}\right]$ there exists some $P$-norm, defined in [12], such that

$$
\left\|H_{1}\right\|_{P}^{\bar{\gamma}}\left\|H_{2}\right\|_{P}^{1-\bar{\gamma}}<1
$$

where

$$
\begin{aligned}
& H_{1}=\left[\begin{array}{c|c}
A+B F & -B F \\
\hline 0 & A-L C A
\end{array}\right] \\
& H_{2}=\left[\begin{array}{c|c}
A & 0 \\
\hline 0 & A-L C A
\end{array}\right] .
\end{aligned}
$$

Proof: It is easy to see that the modified state feedback controller in Eqn. (20) satisfies

$$
\left|u_{k}\right|=\left|F \hat{x}_{k}\right|+\Delta_{k} \geq \Delta_{k}
$$

with equality only holding if $\left|F \hat{x}_{k}\right|=0$. If $\left|F \hat{x}_{k}\right|>0$ then Eqn. (19) is satisfied and $\hat{\gamma}_{k}=\gamma_{k}$, if $\left|F \hat{x}_{k}\right|=0$ then $u_{k}=0$ and the control will not affect the estimation as described in Lemma 4.

We can write the closed loop system as

$$
\begin{aligned}
\mathbf{X}_{k+1} & =H_{\theta(k)} \mathbf{X}_{k}+\Omega_{k} \\
\theta(k) & = \begin{cases}1, & \text { if } \gamma_{k}=1 \\
2, & \text { if } \gamma_{k}=0\end{cases} \\
\Omega_{k} & =\gamma_{k} \operatorname{sgn}\left(F \hat{x}_{k}\right)\left[\begin{array}{l}
B \\
0
\end{array}\right] \Delta_{k} .
\end{aligned}
$$

From above we see the $H_{1}$ matrix will be stable and the $H_{2}$ matrix unstable. Following the definitions of the $P$-norm, $\left\|H_{1}\right\|_{P}<1$ and $\left\|H_{1}\right\|_{P}<\left\|H_{2}\right\|_{P}$.

Define $D_{i}(k)=\prod_{j=i}^{k-1} H_{\theta(j)}$ for $i \leq k-1$ and $D_{k}(k)=$ $I$, where $\theta(j) \in\{1,2\}$ and the multiplication is on the left. For example, $D_{0}(3)=H_{\theta(2)} H_{\theta(1)} H_{\theta(0)}$. Similarly define $E_{i}(k)=\prod_{j=i}^{k-1}\left\|H_{\theta(j)}\right\|_{P}$. We have the following easily verifiable relationships:

1) $D_{i}(k)=D_{j}(k) D_{i}(j)$ and $E_{i}(k)=E_{j}(k) E_{i}(j)$ for any $i \leq j \leq k$

2) $\left\|D_{i}(k)\right\|_{P} \leq E_{i}(k)$

3) $E_{0}(k) \geq E_{i}(k)\left\|H_{1}\right\|_{P}^{i}$ for any $k-1 \geq i \geq 0$

4) $\operatorname{Pr}\left[\lim _{k \rightarrow \infty} E_{0}(k)=\left(\left\|H_{1}\right\|_{P}^{\bar{\gamma}}\left\|H_{2}\right\|_{P}^{1-\bar{\gamma}}\right)^{k}\right]=1$ from the law of large numbers and from Eqn. (21) we see that $\operatorname{Pr}\left[\lim _{k \rightarrow \infty} E_{0}(k)=0\right]=1$ and in fact $E_{0}(k) \rightarrow 0$ as $k \rightarrow \infty$ exponentially fast in $k$. 
Also let $\mathbf{B}=\left\|\left[\begin{array}{c}B \\ 0\end{array}\right]\right\|_{P}$. This allows us to write

$$
\begin{aligned}
\left\|\mathbf{X}_{k}\right\|_{P}= & \left\|D_{0}(k) \mathbf{X}_{0}+\sum_{i=0}^{k-1} D_{i+1}(k) \Omega_{i}\right\|_{P} \\
\leq & \left\|D_{0}(k)\right\|_{P}\left\|\mathbf{X}_{0}\right\|_{P} \\
& +\sum_{i=0}^{k-1}\left\|D_{i+1}(k)\right\|_{P}\left\|\Omega_{i}\right\|_{P} \\
\leq & E_{0}(k)\left\|\mathbf{X}_{0}\right\|_{P}+\sum_{i=0}^{k-1} E_{i+1}(k) \mathbf{B} \Delta_{i} \\
= & E_{0}(k)\left\|\mathbf{X}_{0}\right\|_{P} \\
& +\sum_{i=0}^{k-1} E_{i+1}(k)\left\|H_{1}\right\|_{P}^{i+1} \frac{\mathbf{B} \Delta_{i}}{\left\|H_{1}\right\|_{P}^{i+1}} \\
\leq & E_{0}(k)\left\|\mathbf{X}_{0}\right\|_{P}+\mathbf{B} E_{0}(k) \sum_{i=0}^{k-1} \frac{\Delta_{i}}{\left\|H_{1}\right\|_{P}^{i+1}} \\
= & E_{0}(k)\left(\left\|\mathbf{X}_{0}\right\|_{P}+\frac{2 \mathbf{B}}{|C B|}\|C A\| \delta_{e} \sum_{i=0}^{k-1} K_{i}\right)
\end{aligned}
$$

where

$$
K_{i}=\frac{\left\|(A-L C A)^{i}\right\|}{\left\|H_{1}\right\|_{P}^{i+1}} .
$$

The next step is to prove that $K_{i}$ has a finite upper bound for all $i$. To simplify notation let $A_{1}=A+B F$ and $A_{2}=A-$ $L C A$. Denote $\sigma_{1}=\rho\left(A_{1}\right)$ and $\sigma_{2}=\rho\left(A_{2}\right)$, i.e. the spectral radius of $A_{1}$ and $A_{2}$ respectively, and $\bar{\sigma}=\max \left(\sigma_{1}, \sigma_{2}\right)$. Note since $H_{1}$ is block diagonal with $A_{1}$ and $A_{2}$ on the diagonals we have $\rho\left(H_{1}\right)=\bar{\sigma}$. From pg. 299 of [13] we know that for any matrix norm $\|\cdot\|$ on a matrix $T \in \mathbb{R}^{n \times n}$ we have $\left\|T^{i}\right\| \geq \rho(T)^{i}$. Then we can write

$$
\left\|H_{1}\right\|_{P}^{i+1} \geq\left\|H_{1}^{i+1}\right\|_{P} \geq \rho\left(H_{1}\right)^{i+1}=\bar{\sigma}^{i+1} .
$$

Letting $V_{2}$ be a matrix which can diagonalize $A_{2}$, i.e. $V_{2}^{-1} A_{2} V_{2}=\Lambda_{2}$ with $\Lambda_{2}$ being the diagonal matrix of the eigenvalues of $A_{2}$, we can write

$$
\begin{aligned}
\left\|(A-L C A)^{i}\right\| & =\left\|A_{2}^{i}\right\| \\
& =\left\|V_{2} \Lambda_{2}^{i} V_{2}^{-1}\right\| \\
& \leq\left\|V_{2}\right\|\left\|V_{2}^{-1}\right\|\left\|\Lambda_{2}^{i}\right\| \\
& =\left\|V_{2}\right\|\left\|V_{2}^{-1}\right\| \sigma_{2}^{i}
\end{aligned}
$$

Now let $C_{1}=\frac{\left\|V_{2}\right\|\left\|V_{2}^{-1}\right\|}{\bar{\sigma}}$ and combining the above statements we get

$$
K_{i} \leq C_{1}\left(\frac{\sigma_{2}}{\bar{\sigma}}\right)^{i} \leq C_{1}
$$

In fact, if $\sigma_{1}>\sigma_{2}$ then $\bar{\sigma}=\sigma_{1}$ and $K_{i} \rightarrow 0$ as $i \rightarrow \infty$. Since $(A, B)$ is controllable and $(A, C)$ observable we can make $\sigma_{1}>\sigma_{2}$, this is equivalent to having the observer eigenvalues faster than the controller.

As we only need $K_{i}$ to be upperbounded we can simply use the bound that $K_{i} \leq C_{1}$ for all $i$. Now we return to the
$P$-norm on $\mathbf{X}_{k}$ to get

$$
\begin{aligned}
\left\|\mathbf{X}_{k}\right\|_{P} & \leq E_{0}(k)\left(\left\|\mathbf{X}_{0}\right\|_{P}+\frac{2 \mathbf{B}}{|C B|}\|C A\| \delta_{e} \sum_{i=0}^{k-1} K_{i}\right) \\
& \leq E_{0}(k)\left(\left\|\mathbf{X}_{0}\right\|_{P}+\frac{2 \mathbf{B}}{|C B|}\|C A\| \delta_{e} k C_{1}\right),
\end{aligned}
$$

taking the limit as $k \rightarrow \infty$ we see that from the right hand side we get

$\operatorname{Pr}\left[\lim _{k \rightarrow \infty} E_{0}(k)\left(\left\|\mathbf{X}_{0}\right\|_{P}+k \frac{2 \mathbf{B}}{|C B|}\|C A\| \delta_{e} C_{1}\right)=0\right]=1$

thus $\operatorname{Pr}\left[\lim _{k \rightarrow \infty}\left\|\mathbf{X}_{k}\right\|_{P}=0\right]=1$.

\section{Simulation EXAMPLE}

To illustrate the effectiveness of the estimator algorithm consider the following example,

$$
A=\left[\begin{array}{ll}
1.5 & 0.1 \\
0.3 & 1.3
\end{array}\right], B=\left[\begin{array}{l}
0 \\
1
\end{array}\right], C=\left[\begin{array}{ll}
0 & 1
\end{array}\right] .
$$

The open loop eigenvalues for this system are fairly unstable at $(1.2,1.6)$. The feedback and observer gains are chosen to place the eigenvalues of $A+B F$ and $A-L C A$ at $(0.07,0.08)$ and $(0.05,0.06)$ respectively. This translates into setting the gains to $F=[-20.6,-2.65]$ and $L=[4.6401,0.9984]^{T}$. The initial state and error are assumed to be bounded by $\left\|x_{0}\right\| \leq \sqrt{2}$ and $\left\|e_{0}\right\| \leq \sqrt{2}$. We picked $\bar{\gamma}=0.75$. We simulated the system from random initial conditions and with random realizations of the packet drop sequence.

At first we will assume there is no noise acting on the system. Using the new scheme a typical response profile is shown in Fig. 3. The top plots show the evolution of the state vector and the estimation error. The middle plots show the sequence of $\gamma_{k}$ for this simulation and of $\left(\gamma_{k}-\hat{\gamma}_{k}\right)^{2}$. As can be seen, our estimation algorithm recovers $\hat{\gamma}_{k}=$ $\gamma_{k}$ at every timestep, which allows the estimation error to converge quickly. The control history is plotted in the bottom plots, showing the corrective term decays to zero and $u_{k}$ approaches $F \hat{x}_{k}$.

In Fig. 4 the norm of the state and estimation error are plotted for the algorithm presented in this paper and the other naive schemes described in Section II. The naive schemes that use $\hat{\gamma}_{k}=0$ and $\hat{\gamma}_{k}=\bar{\gamma}$ can be seen to have diverging state and estimation error as expected (they are the two lines that go off the plot after timestep 2). The naive scheme using $\hat{\gamma}_{k}=1$ performs slightly better. As expected the norms of the state and estimation error decrease during the burst of successful packet reception, but during a burst of drops the norms grow very large. In fact they grow to as large as $\left\|e_{19}\right\|=7.62 \times 10^{5}$ and $\left\|x_{21}\right\|=1.95 \times 10^{7}$. So although the naive scheme of $\hat{\gamma}_{k}=1$ may converge almost surely as $k \rightarrow \infty$ for $\bar{\gamma}$ close to unity, it can display this terrible performance during a burst of packet drops.

Though the convergence properties have not been shown to hold in the presence of process and sensor noise, simulations show the algorithm to behave very nicely. We let the process 

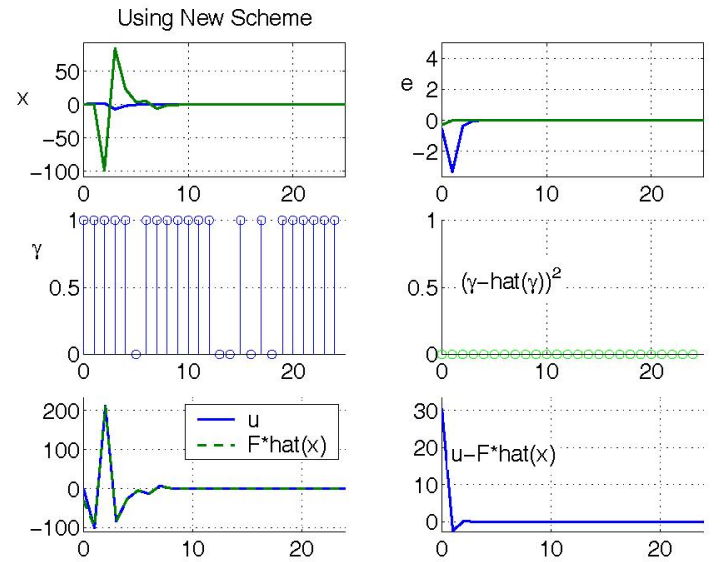

Fig. 3. Simulation results utilizing new estimation scheme.
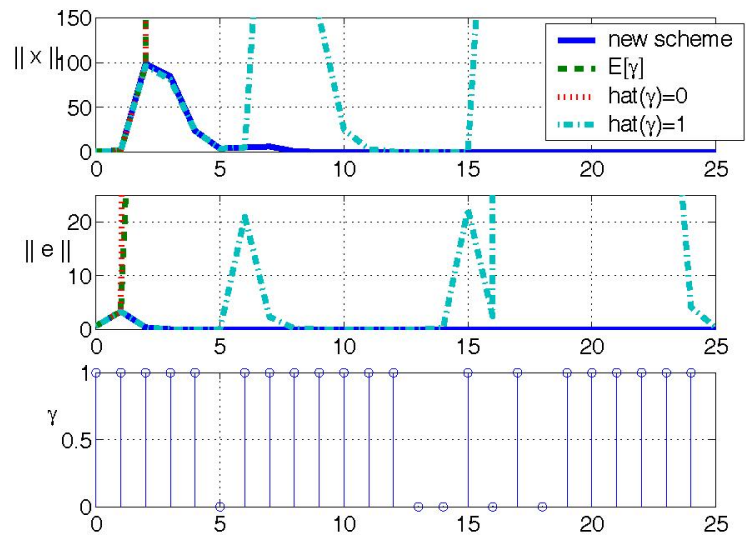

Fig. 4. Comparison of state history for various control-estimation schemes.

noise be bounded by $\left\|w_{k}\right\| \leq \sqrt{2}$ and the sensor noise by $\left\|v_{k}\right\| \leq 1$, thus we get $\delta_{d} \leq 1+\sqrt{2}$ and $\delta_{z} \leq 11.46$. In Fig. 5 we again compare the various schemes.

\section{CONClusions And Future Work}

We presented an approach to control a dynamical system when the control signal is sent from the controller/estimator to the actuators/plant using UDP-like communication protocols, namely without an acknowledgement being sent to the controller to indicate if the plant received the data packet or not. The plant applies the control signal if it is received, otherwise it evolves open loop.

The convergence and closed loop stability proofs required certain restrictions that we would like to relax. The first is that there was no noise. With bounded noise it should be possible to show that state and estimation error remain bounded. Designing a feedback scheme and showing convergence for the general MIMO case would also be a logical next step.

Another extension would be to consider cases where $C B=0$ but $C A^{l} B=0$, that is the input at time $k$ does not appear in the output until time $k+l$. The idea would be to try designing a similar mode observer so that $\gamma_{k}$ can be recovered but not until time $k+l$. This would most likely mean using a state feedback signal delayed $l$ time steps.
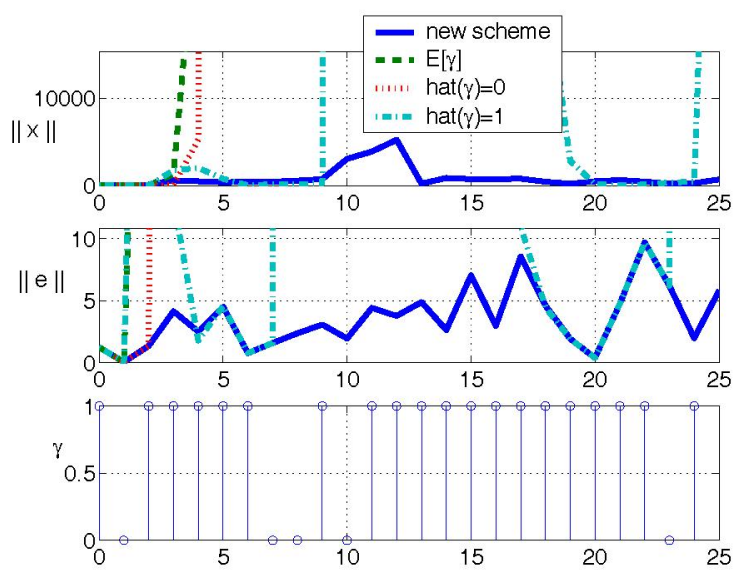

Fig, 5. Comparison of state history for various control-estimation schemes.

Finally, this paper assumed there was no network between the plant/sensors and the controller/esimator. If there were a lossy network between these two units that would mean the estimator would not always have $y_{k}$ available. This would make the recovery of $\gamma_{k}$ most likely impossible when the sensor measurement is lost. Dealing with this situation could be an interesting topic of future research.

\section{ACKNOWLEDGMENTS}

The authors gratefully acknowledge the insightful conversations with Antonis Papachristodoulou.

\section{REFERENCES}

[1] B. Sinopoli, L. Schenato, M. Franceschetti, K. Poolla, M. Jordan, and S. Sastry, "Kalman filtering with intermittent observations," IEEE Transactions on Automatic Control, vol. 49, no. 9, pp. 1453-1464, 2004.

[2] X. Liu and A. Goldsmith, "Kalman filtering with partial observation losses." IEEE Control and Decision, 2004.

[3] L. Shi, M. Epstein, A. Tiwari, and R. M. Murray, "Estimation with information loss: Asymptotic analysis and error bounds," in Intl. Conf. on Decision and Control, 2005.

[4] B. Sinopoli, L. Schenato, M. Franceschetti, K. P. M. Jordan, and S. Sastry, "Optimal control with unreliable communication: the tcp case," American Control Conference, 2005.

[5] B. Sinopoli, L. Schenato, M. Franceschetti, K. Poolla, and S. Sastry, "LQG control with missing observation and control packets," 16th IFAC World Congress, 2005.

[6] - "An LQG optimal linear controller for control systems with packet losses," Conference on Decision and Control, 2005.

[7] A. Alessandri, M. Baglietto, and G. Battistelli, "Receding horizon estimation for switching discrete time linear system," IEEE Conference on Decision and Control, 2004.

[8] V. Gupta, R. M. Murray, and B. Hassibi, "On the control of jump linear markov systems with markov state estimation," American Control Conference, 2003.

[9] M. Babaali and M. Egerstedt, "Aysmptotic observers for discrete-time switched linear systems," IFAC World Congress, 2005.

[10] Y. Fang and K. A. Loparo, "Stochastic stability of jump linear systems," IEEE Transactions on Automatic Control, vol. 47, no. 7, pp. 1204-1208, 2002.

[11] Y. Fang, "A new general sufficient condition for almost sure stability of jump linear systems," IEEE Trans. Automat. Contr, vol. 42, pp. 378-382, March 1997.

[12] L. Shi, M. Epstein, and R. M. Murray, "Towards robust control over a packet dropping network," in Mathematical Theory of Networks and Systems, 2006.

[13] R. A. Horn and C. R. Johnson, Matrix Analysis. Cambridge University Press, 1985. 